\title{
Gemeinsam stark - Ein Erfahrungsbericht zur Zusammenarbeit von Haupt- und Ehrenamtlichen in der Remigius Bücherei, Borken (Westfalen)
}

https://doi.org/10.1515/bfp-2020-0109

Zusammenfassung: Der Erfahrungsbericht berichtet über die Zusammenarbeit von Haupt- und Ehrenamtlichen in der Katholischen Öffentlichen Bücherei St. Remigius in Borken, Nordrhein-Westfalen. Die Strategie der Bibliothek betont das bürgerschaftliche Engagement. Die Freiwilligen sind vor allem im Ausleih- und Benutzungsdienst eingesetzt. Das Management des großen Teams der Freiwilligen erfordert Professionalität.

Schlüsselwörter: Katholische Öffentliche Bücherei; bürgerschaftliches Engagement; Management

Strong Together- Field Report on the Cooperation of Fulltime Staff and Volunteers in the Catholic Public Library St. Remigius in Borken, Germany

Abstract: The field report relates to the cooperation of fulltime staff and volunteers in the Catholic Public Library St. Remigius in Borken, North Rhine-Westphalia. The library's strategy emphasises civic involvement. The volunteers are mainly engaged in lending and user services. The management of the large team of volunteers requires professionalism.

Keywords: Catholic Public Library; civic involvement; management

\section{Steckbrief der Bücherei}

Die Katholische Öffentliche Bücherei St. Remigius in Borken hat sich seit ihrem Gründungsjahr 1852 von einem rein ehrenamtlich geführten Borromäus-Ortsverein zu einer hauptamtlich geleiteten Bibliothek mit aktuell 3,5 Personalstellen sowie einem Auszubildenden (Fachangestellter Medien- und Informationsdienste) entwickelt, ohne das Ehrenamt aus dem Blick zu verlieren.

Mit etwas über 40000 Medieneinheiten ist sie zuständig für die grundlegende Literaturversorgung der nord-

*Kontaktperson: Claudia Heitfeldt, heitfeldt-c@bistum-muenster.de rhein-westfälischen Kreisstadt und hat an sechs Tagen in der Woche (Dienstag bis Sonntag) insgesamt 30 Stunden geöffnet.

Rund 40 Ehrenamtliche leisten ihren Beitrag und sind in unterschiedlichen Aufgabenbereichen und mit verschiedenem Stundeneinsatz tätig.

\section{Strategie und Personal}

Im Jahr 2019 hat die Bücherei an der Fortbildung „Den roten Faden finden - wir entwickeln eine Bibliotheksstrategie“ der Fachstelle für Öffentliche Bibliotheken NRW bei der Bezirksregierung Düsseldorf und des Zentrums für Bibliotheks- und Informationswissenschaftliche Weiterbildung der TH Köln teilgenommen. Ergebnis ist die „Vision 2025“, in der das Büchereiteam als künftige Ausrichtung formuliert: „Remigius Bücherei - Zentrum für Information und Bildung - ,Für dein Leben: gut!““. In dieser Strategie versteht sich die Bücherei als „Zentrum für Information und Begegnung“ sowie als „Partner der religiösen Sozialisation und Bildung für ein ganzheitliches Menschenbild. Sie öffnet Wege zur Selbstfindung und Gotteserfahrung.“ Weiterhin heißt es: „Die Remigius Bücherei fördert bürgerschaftliches Engagement und Ehrenamt. Den haupt- und ehrenamtlichen Mitarbeitern sind christliche Normen und Werte Grundlage ihres Handelns. “1

Auf dem Weg zur Strategieentwicklung wurde auch die aktuelle Personalsituation genauer untersucht.

„Zurzeit arbeiten 40 Ehrenamtliche im Alter von 16 bis 82 Jahren überwiegend im Benutzungsdienst und teilweise in der technischen Medienbearbeitung.

Auch wenn das [...] Diagramm mit 591 Gesamtdienstjahren von einer großen ,Treue‘ zur Remigius Bücherei zeugt, geht die Tendenz eher zu kurz- oder mittelfristigen Verpflichtungen.

In den letzten 10 Jahren ist das Durchschnittsalter des Personals um mehr als 10 Jahre gestiegen. Jugendliche haben zwar Interesse an Büchereiarbeit, sind aber aufgrund der Schulbelastung nur noch sehr selten für den Dienst an Werktagen zu bekommen. So wird das Durchschnittsalter aller Mitarbeiter auf 58 steigen und

1 Hoves (2019) 4. 
das Team zur Hälfte aus Senioren über 60 J. bestehen, wenn es nicht mehr gelingt, jungen Nachwuchs zu bekommen.“2

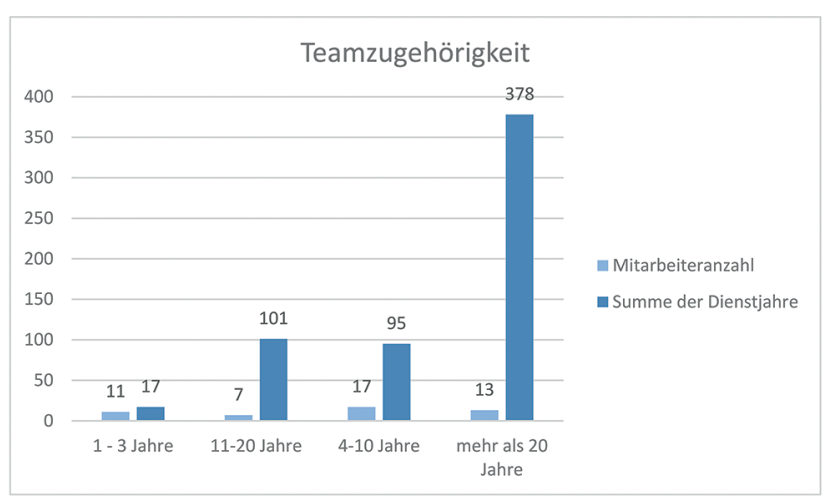

Abb. 1: Teamzugehörigkeit in Jahren

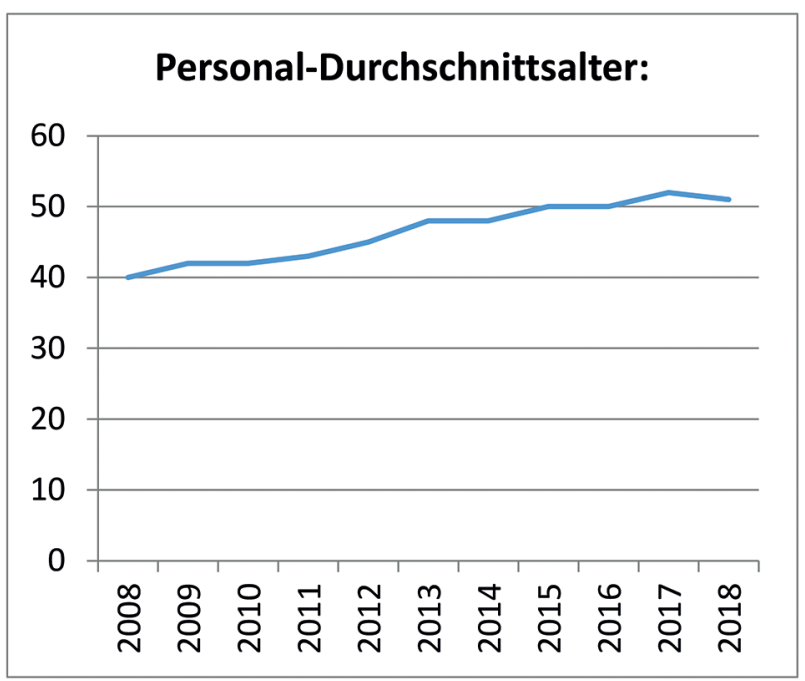

Abb. 2: Personal-Durchschnittsalter

Die verschiedenen Einsatzgebiete des ehrenamtlichen Teams lassen sich wie folgt unterteilen:

- Ausleih- und Benutzungsdienst inklusive Leserberatung (v.a. Leseempfehlungen im Bereich der Schönen Literatur)

- Vorlesen für Kleinkinder

- Leitung des Lese-Erzähl-Cafés für Erwachsene

- Mithilfe bei der technischen Medienbearbeitung

- Betreuung des Makerspace ${ }^{3}$

2 Hoves (2019) 28.

3 Nähere Informationen unter https://b-u-b.de/makerspace/.
Der zeitliche Einsatz reicht von zwei Stunden im Monat (bei Wochenenddiensten), über zwei bis vier Stunden in der Woche bis zu sechs bis acht Stunden bei denjenigen Kolleg^innen, die mehrere Dienste und Einsatzgebiete abdecken.

Der überwiegende Teil der erwachsenen Mitarbeiter*innen der Remigius Bücherei wurde durch Werbung seitens bereits vorhandener Teammitglieder akquiriert. $\mathrm{Zu}$ sätzlich konnten über die Borkener Freiwilligen-Agentur zwei Kolleginnen gewonnen werden, die sich vor allem im Bereich der technischen Medienbearbeitung einsetzen und somit für deutlich schnellere Einarbeitungszeiten bei den neuen Medien sorgen.

Die jugendlichen Mitarbeiter`innen haben größtenteils vorab ein Schulpraktikum in der Bücherei absolviert und sind im Anschluss Teil des ehrenamtlichen Teams geworden.

\section{Management der Ehrenamtlichen}

Die Koordination dieses großen Teams erfordert ein beträchtliches Maß an Kommunikationsfähigkeit und -bereitschaft auf beiden Seiten. Vor allem Änderungen in den Betriebsabläufen, Einführung neuer Medien- oder Veranstaltungsangebote und grundlegende Informationen über die Entwicklung der Remigius Bücherei müssen zeitnah dem gesamten Team mitgeteilt werden. Aber auch kurzfristige Dienstausfälle und die Suche nach Vertretungen sind eine Herausforderung, die in den letzten Jahren durch die Nutzung eines Messenger-Dienstes erheblich vereinfacht wurden. Während zuvor aufwändige Telefonate mit einzelnen Personen nötig waren, kann heute (fast) das komplette Team mit einer Nachricht erreicht werden.

Ein digitaler Kalender, der seit einem knappen Jahr in der Erprobungsphase ist, soll vor allem für die Kolleg*innen, die ausschließlich an den Wochenenden arbeiten, die Übersicht über die Dienstzeiten erleichtern. Hierbei zeigt sich, dass einige Kolleg*innen diesen digitalen Service sehr zu schätzen wissen, während andere lieber „live vor Ort“ in einem zusätzlich geführten, analogen Kalender nachschauen oder telefonisch nachfragen. Diese doppelte Buchführung ist zwar zeitaufwändig, aber so versuchen wir, dem gesamten Team gerecht zu werden und niemanden abzuhängen.

Um das gesamte Team auf dem gleichen Kenntnisstand über die Abläufe im Bücherei-Alltag zu halten, werden zusätzlich bei größeren Veränderungen (neue Systematik, neuer Online-Katalog) interne Schulungen abgehalten. Ein Teil des Teams hat zudem einen BASIS- 
12-Kurs der Büchereifachstelle des Bistums absolviert und so einen besseren Einblick in die Bibliotheksarbeit erhalten. Vor allem der Erfahrungsaustausch mit Kolleg^innen aus anderen KÖB wurde nach der Teilnahme positiv hervorgehoben.

Ein- bis zweimal pro Jahr findet ein Teamtreffen mit allen Mitarbeitenden statt. Dies dient nicht nur dem Austausch aktueller Themen, sondern auch dazu, dass sich die einzelnen Mitarbeiter`innen besser kennenlernen können. Hier werden Vorschläge für den jährlich stattfindenden Betriebsausflug gesammelt sowie die Fahrradtour, die häufig den Besuch anderer Bibliotheken in der Umgebung einschließt und so einen Blick über den Tellerrand ermöglicht.

Beim jährlichen Advents-Brunch erhalten alle Mitarbeiter*innen ein Dankeschön für die geleistete Arbeit, ebenso finden hier die Ehrungen für langjährige Tätigkeiten statt. Durch die eigens eingerichtete Haushaltsstelle „Förderung der Betriebsgemeinschaft“ ist es möglich, diese Anerkennung der Arbeit nicht nur durch einen Handschlag auszudrücken.

Die vielen unterschiedlichen Kompetenzen und Talente des Teams bieten vor allem auch den Nutzer*innen einen Mehrwert, besonders im Bereich der Buchempfehlungen ist das Spektrum bei so vielen „Leseratten“ natürlich deutlich größer und führt auf beiden Seiten $\mathrm{zu}$ einer großen Wertschätzung der Arbeit.

Aber auch der bereitgestellte Geo-Cache ${ }^{4}$ wäre ohne die Anregung und Umsetzung durch eine ehrenamtliche Mitarbeiterin nicht ins Leben gerufen worden.

Beim Aufbau des Makerspace in der Bibliothek geben zwei neu hinzugewonnene Mitarbeiter nicht nur Einführungen in die Welt des 3D-Drucks, sondern haben das hauptamtliche Team auch im Vorfeld mit ihrem Wissen bei der Kaufentscheidung unterstützt und das Gerät in Heimarbeit zusammengebaut.

„Gemeinsam stark“ - dieses Motto bringt es auf den Punkt. Jedes Teammitglied bringt sich nach seinen Fähigkeiten und Möglichkeiten in den Bibliotheksalltag ein, bei besonderen Anlässen (z. B. Änderung der Systematik oder Neuverpackung des CD-Bestandes) ist der Großteil bereit, deutlich mehr als die übliche Zeit und Arbeit in die Verbesserung der Angebote einzubringen.

Bei Fragen oder Problemen wissen alle Ehrenamtlichen, dass sie schwierige Entscheidungen dem hauptamtlichen Personal überlassen können und sollen, so dass das
Konfliktpotenzial mit Nutzer^innen so gering wie möglich gehalten werden kann.

Im Gegenzug finden die Teammitglieder beim hauptamtlichen Personal stets ein offenes Ohr, auch für Fragen oder Probleme außerhalb der eigentlichen Arbeit.

\section{Veränderungen durch die Coronapandemie}

Ein großer Teil des ehrenamtlichen Teams kann aktuell nicht im Publikumsverkehr eingesetzt werden, da sie aufgrund von Vorerkrankungen oder des Alters zur „Risikogruppe“ gehören.

In vielen Gesprächen zeigt sich, dass die regelmäßige Tätigkeit und der Kontakt mit Nutzer^innen dem Team sehr fehlen. Hier offenbart sich mehr als bisher, welch tragende Rolle die soziale Komponente der Arbeit hat. So haben wir uns bemüht, den Mitarbeiter ${ }^{\star}$ innen, die sich in dieser Zeit weiter engagieren möchten, diese Möglichkeit zu geben. So kommen einige Kolleg*innen außerhalb der momentan eingeschränkten Öffnungszeiten, um die zurückgegebenen Medien in die Regale zu räumen und die Regalordnung wieder herzustellen oder übernehmen Teile der technischen Medienbearbeitung.

Digitale Treffen sind aufgrund der z.T. nicht vorhandenen technischen Ausstattung keine Alternative, selbst Zusammenkünfte im Freien können bei der Gruppengröße nicht realisiert werden, da es keine Zusammenkunft im privaten Rahmen ist.

Wir versuchen, den Kontakt über die Messenger-Gruppe aufrecht zu erhalten, einige Mitarbeiter`innen kommen derzeit als Nutzer*innen, wobei deutlich wird, dass die Ausleihe eigentlich nur ein Vorwand ist, um wieder mit bekannten Menschen in Kontakt zu kommen.

Gemeinsam und mit viel Engagement arbeitet das gesamte Team - Haupt- und Ehrenamtliche - auch in diesen veränderten Zeiten daran, das Motto der Bibliotheksstrategie 2025 „Für Dein Leben: gut!“ in die Tat umzusetzen.

4 Nähere Informationen unter https://www.cachewiki.de/wiki/Haup tseite. 


\section{Literaturverzeichnis}

Hoves, Angela (2019): Remigius Bücherei 2015. Borken: Remigius Bücherei 2019. Verfügbar unter https://opacportal.bistum-mue nster.de/borken/Portals/0/bibliotheksstrategie\%20komplett\% 20Sep\%202019.pdf?ver=2020-03-23-094519-173.

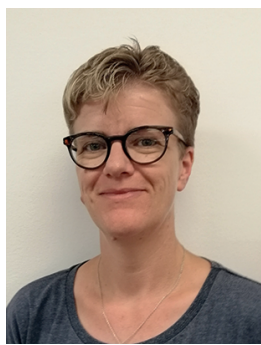

Claudia Heitfeldt

Stellv. Leitung

Remigius Bücherei

Am Vennehof 1

D-46325 Borken

heitfeldt-c@bistum-muenster.de 